\title{
A FUNDAMENTAL DICHOTOMY FOR DEFINABLY COMPLETE EXPANSIONS OF ORDERED FIELDS
}

\author{
ANTONGIULIO FORNASIERO AND PHILIPP HIERONYMI
}

\begin{abstract}
An expansion of a definably complete field either defines a discrete subring, or the image of every definable discrete set under every definable map is nowhere dense. As an application we show a definable version of Lebesgue's differentiation theorem.
\end{abstract}

\section{INTRODUCTION}

Let $\mathbb{K}$ be an expansion of an ordered field $\langle K,<,+, \cdot\rangle$. We say $\mathbb{K}$ is definably complete if every bounded subset of $K$ definable in $\mathbb{K}$ has a supremum in $K$. Such structures were first studied by Miller in 12. A definably complete expansion of ordered field is always real closed. The topology considered here is the usual order topology on $K$ and the product topology on $K^{n}$; all rings are taken with 1 .

The following dichotomy is the main result of the paper.

Theorem A. Let $\mathbb{K}$ be definably complete. Then either

(I) $f(D)$ is nowhere dense for every definable discrete set $D \subseteq K^{n}$ and every definable function $f: K^{n} \rightarrow K$, or

(II) $\mathbb{K}$ defines a discrete subring.

This result is a generalization of [6. Theorem 1.1] from expansions of the real field to arbitrary definably complete expansions of ordered fields. The two cases in Theorem $\mathrm{A}$ are indeed exclusive. It is easy to check that a definable subring has to be unbounded and that its set of quotients is dense in $K$. By definable completeness, the positive elements of a definable discrete subring of $\mathbb{K}$ form a model of first-order Peano arithmetic; in $\$ 5$ we will see that they even form a model of second-order Peano arithmetic (seen as a first-order theory). Hence Theorem A separates the class of definably complete expansions of ordered fields into two very distinct categories.

The significance of Theorem A comes from its use as a tool to prove statements about arbitrary definably complete expansions of ordered fields. In order to show that a statement holds for all such structures, it is now enough to consider structures having either property (I) or (II) from Theorem A. In the case when a discrete subring is definable, proofs from second-order arithmetic often transfer easily to these structures. On the other hand, if a structure satisfies property (I), techniques and

Date: July 18, 2018.

2000 Mathematics Subject Classification. Primary 03C64.

A version of this paper will appear in the Journal of Symbolic Logic. The first author was supported by Italian FIRB 2010 "New advances in the Model Theory of exponentiation". The second author was partially supported by NSF grant DMS-1300402 and by UIUC Campus Research Board award 13086. 
ideas from the study of o-minimality and related tameness notions can sometimes be applied. As an application of this new proof strategy we present the following definable analogue of Lebesgue's differentiation theorem, answering a question of Miller from [12].

Theorem B. Let $\mathbb{K}$ be definably complete and let $f: K \rightarrow K$ be definable and monotone. Then $f$ is differentiable on a dense subset of $K$.

Notation. For the rest of the paper, let $\mathbb{K}$ denote a definable complete expansion of an ordered field $\langle K,<,+, \cdot\rangle$. We say a set is definable if it is definable in $\mathbb{K}$ with parameters from $K .\langle a, b\rangle$ is the ordered pair with elements $a$ and $b$. Given a subset $X$ of $K^{n} \times K^{m}$ and $a \in K^{n}$, we denote the set $\{b:\langle a, b\rangle \in X\}$ by $X_{a}$. As said before, all rings are taken with 1.

Acknowledgements. The authors would like to thank Lou van den Dries and the anonymous referees for closely reading the paper and for their valuable comments.

\section{Facts about Definable COMPlete FiElds}

In this section we recall several facts about definably complete expansions of ordered fields. For more details and background, see [12. The following fact is immediate from definable completeness.

Fact 1. Let $Y \subseteq K$ be non-empty closed and definable. Then $Y$ contains a minimum (a maximum) iff $Y$ is bounded from below (from above).

Fact 2 ([12, Lemma 1.9]). Let $Y \subseteq K^{2}$ be definable such that $Y_{a}$ is closed and bounded and $Y_{a} \supseteq Y_{b} \neq \emptyset$ for every $a, b \in K$ with $a<b$. Then $\bigcap_{a \in K} Y_{a} \neq \emptyset$.

Definition 3. Let $D \subseteq K$ be definable, closed and discrete and let $d \in D$. If $d$ is not the maximum of $D$, we say the minimum of $D_{>d}$ is the successor of $d$ in $D$, written $s_{D}(d)$.

Note that the minimum in the previous definition exists by Fact 1 .

Fact 4. Let $D \subseteq K$ be definable, closed and discrete. If $D$ has a minimum (a maximum), so has every definable subset of $D$.

Definition 5. A subset $A \subseteq K^{n}$ is called pseudo-finite if it is definable, closed, bounded and discrete. We call $A$ at most pseudo-enumerable if there exists a definable closed discrete set $D \subset K_{\geq 0}$ and a definable function $f: D \rightarrow K^{n}$ such that $f(D)=A$.

The notion of a pseudo-finite set was introduced in [4] and the notion of at most pseudo-enumerable in [3].

Fact 6 ([3, Main Theorem]). If $A \subseteq K^{n}$ is at most pseudo-enumerable, then it has no interior.

Fact 7 (4 Lemma 2.22]). Let $D \subseteq K^{n}$ be pseudo-finite and let $f: D \rightarrow K^{m}$ be a definable function. Then $f(D)$ is pseudo-finite. In particular, $f$ achieves a minimum and a maximum on $D$.

Fact 8 ([3. Lemma 4.14]). Every definable discrete subset of $K^{n}$ is at most pseudoenumerable. 
Fact 8 simplifies our task to prove Theorem A considerably. To establish Theorem A, it is now enough to show that whenever $\mathbb{K}$ defines a closed and discrete set $D \subseteq K_{\geq 0}$ and a function $f: D \rightarrow K$ with $f(D)$ somewhere dense, then $\mathbb{K}$ defines a discrete subring.

Definition 9. A definable family $\left(X_{t}: t \in D\right)$ is at most pseudo-enumerable if its index set $D$ is at most pseudo-enumerable.

The following fact was implicitly proved in 3 . For the reader's convenience, we have included a proof here.

Fact 10. (1) The union of an at most pseudo-enumerable family of discrete sets is at most pseudo-enumerable.

(2) Let $\left(X_{t}: t \in K\right)$ be a definable increasing family of discrete subsets of $K^{n}$. Then $\bigcup_{t \in K} X_{t}$ is at most pseudo-enumerable.

Proof. Statement (1) is [3, Corollary 4.16]. We now consider (2). By 4, Theorem $3.3], \mathbb{K}$ either defines a discrete, closed and unbounded set, or every discrete set definable in $\mathbb{K}$ is pseudo-finite. We now handle the cases separately. If every discrete definable set in $\mathbb{K}$ is pseudo-finite, then each $X_{t}$ is pseudo-finite. By 4 , Theorem 3.3] $\bigcup_{t \in K} X_{t}$ itself is pseudo-finite. Now suppose that there exists $D \subseteq$ $K_{>0}$ definable, discrete, closed and unbounded. Since $\left(X_{t}: t \in K\right)$ is increasing and $D$ is unbounded, $\bigcup_{t \in K} X_{t}=\bigcup_{t \in D} X_{t}$. By (1) applied to the family $\left(X_{t}: t \in D\right)$, $\bigcup_{t \in K} X_{t}$ is at most pseudo-enumerable.

\section{NATURAL FRAGMENTS AND ASYMPtotic EXTRACTION}

In this section we generalize the idea of asymptotic extraction, first introduced by Miller in [13, p. 1484], to definably complete fields. Since the original approach is not strong enough to yield the desired results, we adjust the method developed in [7, Lemma 1] to extract larger and larger fragments of the natural numbers.

Definition 11. Let $D$ be a definable, closed and discrete subset of $K_{>0}$. We say that $D$ has step 1 if, for every $d \in D$ with $d \neq \max (D), s_{D}(d)=d+1$. We say that $D$ is a natural fragment if it is either empty, or if $D$ has step 1 and $0 \in D$.

Lemma 12. Let $D$ and $E$ be natural fragments. Then either $D \subseteq E$ or $E \subseteq D$.

Proof. Suppose not. Let $d=\min (D \backslash E \cup E \backslash D)$. Without loss of generality, assume $d \in D$. Since $0 \in D \cap E, d>0$. Since $D$ is a natural fragment, $d-1 \in D$. Since $d$ was chosen to be minimal, $d-1 \in E$ as well. Since $d \notin E, d-1$ has to be the maximum of $E$. Since $D \cap[0, d-1]=E \cap[0, d-1]$ by minimality of $d$, we have $E \subseteq D$.

Corollary 13. Let $\left(X_{t}: t \in I\right)$ be a definable family such that $X_{t}$ is a natural fragment for each $t \in I$. Then $\bigcup_{t \in I} X_{t}$ is a natural fragment.

It is worth noting that by Lemma 12 the union of all natural fragments, although not necessarily definable, is closed, discrete, contains 0 and has step 1.

Definition 14. Let $D$ be a definable, closed and discrete subset of $K_{\geq 0}$ and $\varepsilon \in$ $K_{>0}$. We say that $D$ is an $\varepsilon$-natural fragment if

(1) $\left|s_{D}(d)-(d+1)\right|<\varepsilon$ for every $d \in D$ with $d \neq \max D$,

(2) $\operatorname{dist}(D, 0)<\varepsilon$. 
For $a \in K_{\geq 0}$, we say $D$ is an $\varepsilon$-natural fragment close to $a$ if $\operatorname{dist}(D, a)<\varepsilon$.

The next Lemma shows that the property of being an $\varepsilon$-natural fragment for some $\varepsilon$ is preserved under small changes.

Lemma 15. Let $\varepsilon \in K_{>0}$ with $\varepsilon<\frac{1}{4}$, let $D$ be a $\varepsilon$-natural fragment close to $a$ and let $f: D \rightarrow(-\varepsilon, \varepsilon)$ be a definable function. Then

$$
E:=\{d+f(d): d \in D\}
$$

is a $3 \varepsilon$-natural fragment close to $a$.

Proof. Set $g(d):=d+f(d)$ for $d \in D$. It is immediate that (2) holds for $E$ and $3 \varepsilon$, since it holds for $D$ and $\varepsilon$. Since (1) holds for $D$ and $\varepsilon$ and $\varepsilon<1 / 4$, $g\left(s_{D}(d)\right)=s_{E}(g(d))$ for every $d \in D$ with $d \neq \max D$. Moreover, for every $d \in D$ with $d \neq \max D$,

$$
\left|s_{E}(g(d))-g(d)-1\right|<2 \varepsilon+\left|s_{D}(d)-d-1\right|<3 \varepsilon .
$$

Hence (1) holds for $E$ and $3 \varepsilon$. Hence $E$ is a $3 \varepsilon$-natural fragment close to $a$.

Definition 16. Let $\left(Y_{t}: t \in I\right)$ be a definable family of subsets of $K$. The natural fragment extracted from $\left(Y_{t}: t \in I\right)$ is the set of $d \in K_{\geq 0}$ such that for every $\varepsilon \in K_{>0}$ there exists $t \in I$ such that $Y_{t}$ is an $\varepsilon$-natural fragment close to $d$.

It is not obvious that the object defined in the previous definition is a natural fragment in sense defined before. The following Lemma establishes that this is indeed the case.

Lemma 17. Let $\left(Y_{t}: t \in I\right)$ be a definable family of subsets of $K$. Then the natural fragment extracted from $\left(Y_{t}: t \in I\right)$ is a natural fragment.

Proof. Let $D$ be the natural fragment extracted from $\left(Y_{t}: t \in I\right)$. Since the empty set is a natural fragment, we reduce to the case that $D$ is non-empty. It follows easily from the definitions that $0 \in D$ whenever $D$ is non-empty.

For $d \in D$ consider the definable set $E_{d}$ consisting of all $e \in K$ with $e \leq d$ such that for every $\varepsilon \in K_{>0}$ there exists $t \in I$ such that $Y_{t}$ is an $\varepsilon$-natural fragment close to $d$ and $\operatorname{dist}\left(Y_{t}, e\right)<\varepsilon$. Note that $d \in E_{d}$ and $E_{d} \subseteq D$. Hence $\bigcup_{d \in D} E_{d}=D$. Thus by Corollary 13 it is enough to show that each $E_{d}$ is a natural fragment.

Let $d \in D$. We first show that $e+1 \in E_{d}$ for every $e \in E_{d}$ with $e \leq d-1$. Let $\varepsilon \in K$ such that $0<\varepsilon<1$. Take $t \in I$ such that $Y_{t}$ is a $\frac{\varepsilon}{2}$-natural fragment close to $d$ and $\operatorname{dist}\left(Y_{t}, e\right)<\frac{\varepsilon}{2}$. Let $y \in Y_{t}$ be such that $|e-y|<\frac{\varepsilon}{2}$. Since $e \leq d-1$ and $\operatorname{dist}\left(Y_{t}, d\right)<\frac{\varepsilon}{2}, y$ is not the maximum of $Y_{t}$. Then

$$
\begin{aligned}
\left|s_{Y_{t}}(y)-(e+1)\right| & =\left|s_{Y_{t}}(y)+y-y-(e+1)\right| \\
& \leq\left|s_{Y_{t}}(y)-y-1\right|+|y-e|<\varepsilon .
\end{aligned}
$$

Hence $\operatorname{dist}\left(Y_{t}, e+1\right)<\varepsilon$. Thus $e+1 \in E_{d}$. Similarly, we can show that $e-1 \in E_{d}$ for every $e \in E_{d}$ with $e \geq 1$.

Consider

$$
B:=\left\{e \in E_{d}:[e, e+1) \cap E_{d}=\{e\}\right\} .
$$

Note that $B$ is closed and discrete and $d \in B$. We will now show that $B$ is a natural fragment. It is easy to see that $0 \in B$. Let $e \in B$ and suppose $e \leq d-1$. Then 
$e+1 \in E_{d}$. Towards a contradiction assume $e+1 \notin B$. Then there is $l \in E_{d}$ such that $e+1<l<e+2$. Since $l \geq 1$ and $l \in E_{d}$, we have $l-1 \in E_{d}$ with $e<l-1<e+1$. Hence $e \notin B$, a contradiction.

It is left to show that $E_{d}=B$. Towards a contradiction suppose there is $e \in E_{d} \backslash B$. By Fact 4 there is a maximal $l \in B$ smaller than $e$. Since $l<d, l+1 \in B$. Since $l \in B$ and $e \notin B, l+1<e$. A contradiction against the maximality of $l$. Hence $E_{d}=B$.

It is worth pointing out that until this point only the additive structure of $\mathbb{K}$ has been used.

Proposition 18. Let $D$ be an unbounded natural fragment. Then $\langle D,+, \cdot,<\rangle$ is a model of first-order Peano arithmetic. Moreover, $D \cup-D$ is a definable discrete subring of $\mathbb{K}$.

Proof. Let $D$ be an unbounded natural fragment. We first show that $D$ is closed under addition. Suppose not. By Fact 4 we can take $d \in D$ minimal such that there is $e \in D$ with $d+e \notin D$. Clearly, $d \neq 0$. Since $d$ is minimal, $(d-1)+(e+1) \in D$. A contradiction. Hence $D$ is closed under addition.

Now suppose that $D$ is not closed under multiplication. Again take $d \in D$ minimal such that there is $e \in D$ with $d \cdot e \notin D$. Clearly, $d \neq 0$. By minimality of $d$, $(d-1) \cdot e \in D$ and hence $(d-1) \cdot e+e \in D$. Hence $D$ is closed under multiplication. Since every definable subset of $D$ has a minimum by Fact $4 .\langle D,+, \cdot,<\rangle$ satisfies the first-order induction axiom. Hence $\langle D,+, \cdot,<\rangle$ is a model of first-order Peano arithmetic.

Now set $Z:=D \cup-D$. It follows immediately that $\langle Z,+, \cdot\rangle$ is a discrete subring of $\mathbb{K}$.

\section{Best approximations and the proof of Theorem A}

Let $\mathbb{K}$ be a definably complete expansion of an ordered field that defines a closed and discrete set $D \subseteq K_{\geq 0}$ and a function $f: D \rightarrow K$ with $f(D)$ somewhere dense. In order to establish Theorem $\mathrm{A}$, it is enough by Fact 8 to define a discrete subring. By Proposition 18 it suffices to define an unbounded natural fragment. After composing $f$ with a semialgebraic function we can assume that $f(D)$ is dense in $(0,1)$. First several definitions related to this function $f$ will be introduced. These definitions were first used for expansions of $\mathbb{R}$ by Hieronymi and Tychonievich in $[9]$.

Definition 19. Let $c \in(0,1)$. We say $d \in D$ is a best approximation of $c$ from the left if $f(d)<c$ and

$$
f\left(D_{<d}\right) \cap(f(d), c)=\emptyset .
$$

We write $L_{c}$ for the set of best approximations of $c$ from the left. Similarly, we say $d \in D$ is a best approximation of $c$ from the right if $f(d)>c$ and

$$
f\left(D_{<d}\right) \cap(c, f(d))=\emptyset .
$$

and write $R_{c}$ for the set of best approximations of $c$ from the right.

For $d \in D$, we write

$$
L_{c, d}:=L_{c} \cap[0, d] \text { and } R_{c, d}:=R_{c} \cap[0, d],
$$


and

$$
l_{c, d}:=\left\{\begin{array}{ll}
f\left(\max L_{c, d}\right), & \text { if } L_{c, d} \neq \emptyset ; \\
0, & \text { otherwise, }
\end{array} \text { and } r_{c, d}:= \begin{cases}f\left(\max R_{c, d}\right), & \text { if } R_{c, d} \neq \emptyset ; \\
1, & \text { otherwise. }\end{cases}\right.
$$

Since $D$ is closed and discrete, both $L_{c}$ and $R_{c}$ are closed and discrete by Fact 4 Since $D_{\leq d}$ is pseudo-finite, so is $f\left(D_{\leq d}\right)$ by Fact 7 . Hence both $L_{c}$ and $R_{c}$ are non-empty. It is easy to check that by density of $f(D)$ both $L_{c}$ and $R_{c}$ are unbounded and $c=\sup f\left(L_{c}\right)=\inf f\left(R_{c}\right)$ for $c \in(0,1)$. Moreover $L_{c, d}$ and $R_{c, d}$ are pseudo-finite and the maximum used in the above definition actually exists. It also worth pointing out that this implies $l_{c, d}<c<r_{c, d}$.

Lemma 20. Let $a \in(0,1) \backslash f(D)$ and $d \in D$. Then $L_{a, d}=L_{b, d}$ and $R_{a, d}=R_{b, d}$ for every $b \in\left(l_{a, d}, r_{a, d}\right)$.

Proof. By definition of $l_{a, d}$ and $r_{a, d}$,

$$
f\left(D_{\leq d}\right) \cap\left(\left(l_{a, d}, a\right) \cup\left(a, r_{a, d}\right)\right)=\emptyset .
$$

Since $a \notin f(D), f\left(D_{\leq d}\right) \cap\left(l_{a, d}, r_{a, d}\right)=\emptyset$. Hence for all $b \in\left(l_{a, d}, r_{a, d}\right)$

$$
\left\{e \in D_{\leq d}: f(e)<b\right\}=\left\{e \in D_{\leq d}: f(e)<a\right\}
$$

and

$$
\left\{e \in D_{\leq d}: f(e)>b\right\}=\left\{e \in D_{\leq d}: f(e)>a\right\} .
$$

Thus $L_{a, d}=L_{b, d}$ and $R_{a, d}=R_{b, d}$.

The strategy for the rest of proof is as follows. We will introduce a definable family using the notions introduced above. Then it will be shown that the natural fragment extracted from this family is unbounded. The idea how to show the last statement is the following: suppose there is $b \in K, \varepsilon \in K_{>0}$ and a suitable semialgebraic function $g$ such that the image of a definable subset of $L_{b, d} \times\{b\} \times R_{b, d}$ is an $\varepsilon$-natural fragment close to some $n \in K$. By Lemma 20, the set $L_{b, d}$ and $R_{b, d}$ do not change on an interval around $b$. Being careful with the definitions we will use this statement to show that we can find an element $c$ close to $b$ and $d^{\prime} \in D$ such that the image of a definable subset of $L_{c, d^{\prime}} \times\{c\} \times R_{c, d^{\prime}}$ under $g$ is a $6 \varepsilon$-natural fragment close to $n+1$.

Let $g: K^{3} \rightarrow K$ be

$$
g(a, b, c):= \begin{cases}\frac{c-a}{b-a} & \text { if } a<b<c \\ 0 & \text { otherwise. }\end{cases}
$$

We will now define a family of definable sets from which we extract an unbounded natural fragment. Let $\langle a, b\rangle \in(0,1)^{2}$ and $d \in D$. Define

$$
Y_{a, b, d}:=\{0\} \cup\left\{g\left(l_{b, e}, b, r_{b, e}\right): e \in L_{a, d}\right\} .
$$

Let $J$ be the set of $\langle a, b, d\rangle \in((0,1) \backslash f(D))^{2} \times D$ such that the map $e \mapsto g\left(l_{b, e}, b, r_{b, e}\right)$ is strictly increasing on $L_{a, d}$. Note that $\left(Y_{a, b, d}\right)_{\langle a, b, d\rangle \in J}$ is indeed a definable family.

Lemma 21. Let $\langle a, b, d\rangle \in J, c \in(0,1), u \in K$ and $\varepsilon \in K_{>0}$ with $\varepsilon<\frac{1}{4}$. Then

(i) if $L_{a, d}=L_{c, d}$, then $Y_{a, b, d}=Y_{c, b, d}$.

(ii) if $Y_{a, b, d}$ is an $\varepsilon$-natural fragment close to $u$, then there is an interval $I$ around $b$ such that for all $c \in I \backslash f(D)$, we have $\langle a, c, d\rangle \in J$ and $Y_{a, c, d}$ is a $3 \varepsilon$-natural fragment close to $u$. 
Proof. Statement (i) is immediate from the definitions. For (ii) let $I_{0}$ be the interval $\left(l_{b, d}, r_{b, d}\right)$. By Lemma20 and $b \notin f(D), L_{c, d}=L_{b, d}$ and $R_{c, d}=R_{b, d}$ for every $c \in I_{0}$. For each $e \in L_{a, d}$ let $I_{e}$ be the maximal open subinterval of $I_{0}$ containing $b$ such that for each $c \in I_{e}$

$$
\left|g\left(l_{c, e}, c, r_{c, e}\right)-g\left(l_{b, e}, b, r_{b, e}\right)\right|<\varepsilon .
$$

This choice is possible, since $g$ is continuous in the second coordinate and $l_{c, e}=l_{b, e}$ and $r_{c, e}=r_{b, e}$ for every $c \in I_{0}$. Note that the maps $e \in L_{a, d} \mapsto \sup I_{e}$ and $e \in L_{a, d} \mapsto \inf I_{e}$ are definable. Hence by Fact 7 both functions have a maximum and a minimum on $L_{a, d}$. Hence there is $e_{1}, e_{2} \in L_{a, d}$ such that

$$
\bigcap_{e \in L_{a, d}} I_{e}=\left(\inf I_{e_{1}}, \sup I_{e_{2}}\right) \text {. }
$$

Let $I$ be this open interval. Since $b \in I, I$ is non-empty. Since $\varepsilon<\frac{1}{4}$ and $Y_{a, b, d}$ is an $\varepsilon$-natural fragment, the map $e \mapsto g\left(l_{c, e}, c, r_{c, e}\right)$ is strictly increasing on $L_{a, d}$ for every $c \in I \backslash f(D)$. Hence $\langle a, c, d\rangle \in J$ for all such $c$.

Let $c \in I$. Let $k: Y_{a, b, d} \rightarrow(-\varepsilon, \varepsilon)$ map 0 to 0 and $g\left(l_{b, e}, b, r_{b, e}\right)$ to

$$
g\left(l_{c, e}, c, r_{c, e}\right)-g\left(l_{b, e}, b, r_{b, e}\right) .
$$

This function is well-defined, since $\langle a, b, d\rangle \in J$ and hence $e \mapsto g\left(l_{b, e}, b, r_{b, e}\right)$ is strictly increasing on $L_{a, d}$. By definition

$$
Y_{a, c, d}=\left\{y+k(y): y \in Y_{a, b, d}\right\} .
$$

By definability of $k$, 4.1) and Lemma 15 this set is a $3 \varepsilon$-natural fragment.

Theorem 22. The natural fragment extracted from $\left(Y_{a, b, d}:\langle a, b, d\rangle \in J\right)$ is unbounded.

Proof. Let $F$ be the natural fragment extracted from $\left(Y_{a, b, d}:\langle a, b, d\rangle \in J\right)$. We first show that $F$ is non-empty. It is enough to find for every $\varepsilon \in K_{>0}$ a triple $\langle a, b, d\rangle \in J$ such that $Y_{a, b, d}$ is an $\varepsilon$-natural fragment up to 1 . Let $d \in D$ be the smallest element of $D$. Take $a, b \in[0,1] \backslash f(D)$ such that

$$
0<\frac{f(d)}{1+\varepsilon}<b<f(d)<a<1 \text {. }
$$

Then $L_{a, d}=\{d\}, L_{b, d}=\emptyset$ and $R_{b, d}=\{d\}$. Hence $l_{b, d}=0$ and $r_{b, d}=f(d)$. Thus

$$
\left|g\left(l_{b, d}, b, r_{b, d}\right)-1\right|=\left|\frac{f(d)}{b}-1\right|<\varepsilon
$$

Hence $Y_{a, b, d}=\left\{0, \frac{f(d)}{b}\right\}$ is an $\varepsilon$-natural fragment up to 1 .

Now towards a contradiction suppose that $F$ is bounded. Let $n$ be the maximum of $F$. We will establish a contradiction against the maximality of $n$. For this, it is enough to construct for every $\varepsilon \in K_{>0}$ a triple $\langle a, b, d\rangle \in J$ such that $Y_{a, b, d}$ is an $\varepsilon$-natural fragment close to $n+1$.

Let $\varepsilon \in K_{>0}$. Since $n$ is in the natural fragment extracted from $\left(Y_{a, b, d}:\langle a, b, d\rangle \in\right.$ $J)$, there is $\langle u, v, e\rangle \in J$ such that $Y_{u, v, e}$ is an $\frac{\varepsilon}{6}$-natural fragment close to $n$. Let $I$ be the interval around $v$ given by Lemma 21(ii) such that for every $w \in I \backslash f(D)$, 
$Y_{u, w, e}$ is an $\frac{\varepsilon}{2}$-natural fragment close to $n$ and $\langle u, w, e\rangle \in J$.

Let $d_{0}$ be an element of $D_{\geq e}$ such that there are $e_{1}, e_{2} \in D$ with $e_{1}, e_{2} \leq d_{0}$, $f\left(e_{1}\right)<f\left(e_{2}\right)$, and

$$
\left(f\left(e_{1}\right), f\left(e_{2}\right)\right) \subseteq I
$$

Such an element exists because of the density of $f(D)$. Choose $a \in K \backslash f(D)$ such that $l_{u, e}<a$ and

$$
\left(l_{u, e}, a\right) \cap f\left(D_{\leq d_{0}}\right)=\emptyset .
$$

We can find such an element because $f\left(D_{\leq d_{0}}\right)$ is pseudo-finite and $f(D)$ does not have interior by Fact 6 . Now let $d \in D$ be the smallest element in $D_{\geq d_{0}}$ with

$$
f(d) \in\left(l_{u, e}, a\right)
$$

Then $L_{a, d}=L_{u, e} \cup\{d\}$.

It is left pick to $b$. First take $e_{1}, e_{2} \in D_{\leq d}$ such that $f\left(e_{1}\right)<f\left(e_{2}\right)$,

$$
\left(f\left(e_{1}\right), f\left(e_{2}\right)\right) \subseteq I \quad \text { and } \quad\left(f\left(e_{1}\right), f\left(e_{2}\right)\right) \cap f\left(D_{\leq d}\right)=\emptyset .
$$

This choice is possible, because $f\left(D_{\leq d}\right)$ is pseudo-finite. Now pick $b \in\left(f\left(e_{1}\right), f\left(e_{2}\right)\right)$ such that $b \notin f(D)$ and

$$
\left|g\left(f\left(e_{1}\right), b, f\left(e_{2}\right)\right)-(n+1)\right|<\frac{\varepsilon}{2} .
$$

By our choice of $b, f\left(e_{1}\right)=l_{b, d}$ and $f\left(e_{2}\right)=r_{b, d}$. Since $b \in I \backslash f(D), Y_{u, b, e}$ is a $\frac{\varepsilon}{2}$-natural fragment close to $n$ and $\langle u, b, e\rangle \in J$. Since $L_{u, e}=L_{a, e}$ by choice of $a$, we have that $\langle a, b, e\rangle \in J$ and $Y_{u, b, e}=Y_{a, b, e}$ by Lemma 21(i). Hence $Y_{a, b, e}$ is a $\frac{\varepsilon}{2}$-natural fragment close to $n$. If $Y_{a, b, e}$ is a $\frac{\varepsilon}{2}$-natural fragment close to $n+1$, then $Y_{a, b, e}$ is also a $\varepsilon$-natural fragment close to $n+1$ and hence we are done. Thus from now on we can assume that $Y_{a, b, e}$ is not a $\frac{\varepsilon}{2}$-natural fragment close to $n+1$. Then

$$
\left|\max Y_{a, b, e}-n\right|<\frac{\varepsilon}{2} \text {. }
$$

Now set $z:=g\left(l_{b, d}, b, r_{b, d}\right)$. Since $L_{a, d}=L_{u, e} \cup\{d\}$, we have

$$
Y_{a, b, d}=Y_{a, b, e} \cup\{z\} \text {. }
$$

Then by (4.2) and (4.3)

$$
\left|z-\max Y_{a, b, e}-1\right| \leq|z-(n+1)|+\left|\max Y_{a, b, e}-n\right|<\frac{\varepsilon}{2}+\frac{\varepsilon}{2}=\varepsilon .
$$

Hence $Y_{a, b, d}$ is an $\varepsilon$-natural fragment close to $n+1$.

The proof of the above theorem can be easily adapted to show that, for every pseudo-finite set $F \subseteq K_{\geq 1},\{0\} \cup F$ can be approximated arbitrarily close by some $Y_{a, b, d}$ : i.e., for every $\varepsilon>0$ there exists $\langle a, b, d\rangle \in J$ such that $\operatorname{dist}\left(\{0\} \cup F, Y_{a, b, d}\right)<\varepsilon$.

As shown in Proposition 18, if $D$ is the unbounded natural fragment extracted in Theorem 22, then $D \cup-D$ is a definable discrete subring of $\mathbb{K}$, and Theorem A follows. 


\section{UnRESTRAined DC STRUCTURES}

Definition 23. We say that $\mathbb{K}$ is unrestrained if it defines a discrete subring, otherwise, we say that $\mathbb{K}$ is restrained.

We claim that unrestrained structures are the same as model of second-order arithmetic, in a sense that we will make precise. The result of this section will be used in 97

First, we make precise what we mean by model of second-order arithmetic; as a background reference we use [19], especially its $\oint 1$. Let $L_{2}:=\langle N, D ; 0,1,+, \cdot,<\rangle$ be the (first-order!) 2-sorted language of second-order arithmetic, with a sort $N$ for ("natural") numbers (which will be denoted by lowercase letters) and one sort $D$ for sets (which will be denoted by uppercase letters), with number constants 0 and 1 , binary operations + and $\cdot$ and a binary relation $<$ on numbers, and a binary relation $\in$ between numbers and sets. Let $L$ be an arbitrary expansion of $L_{2}$ (here we differ from [19, where only structures in the language $L_{2}$ are considered); notice that we allow extra function symbols and predicates that involve the sort $D$ and not only new function and relation symbols on $N$. A model of second-order arithmetic is a (again, first-order) $L$-structure $\mathcal{N}:=\langle N, D ; 0,1,+, \cdot,<, \ldots\rangle$ satisfying the following axioms:

Basic axioms: $\langle N,+, 0,1,+, \cdot,<\rangle$ is the positive cone of a discrete linearly ordered $\operatorname{ring} Z$;

\section{Extension axiom:}

$$
\forall X \forall Y(\forall n(n \in X \leftrightarrow n \in Y) \rightarrow X=Y) ;
$$

\section{Induction axiom:}

$$
\forall X((0 \in X \& \forall n(n \in X \rightarrow n+1 \in X)) \rightarrow X=N)
$$

\section{Comprehension scheme:}

$$
\exists X \forall n(n \in X \leftrightarrow \phi(n)),
$$

where $\phi(n)$ is any $L$-formula in which $X$ does not occur freely.

Remember that we view second-order arithmetic as a theory in first-order logic; thus, the theory will have models besides the standard one, given by $\mathbb{N}$ and all its subsets.

5.1. From unrestrained structures to models of arithmetic. First, we show how to transform an unrestrained structure into a model of second-order arithmetic.

For the rest of this subsection, let $\mathbb{K}$ be unrestrained. Let $Z$ be a definable discrete subring of $\mathbb{K}$. Note that $Z$ is the unique subring with that property: if $Z^{\prime}$ were another discrete definable subring of $K$, one considers the minimum positive element of $Z \Delta Z^{\prime}$ and easily reaches a contradiction against $Z \neq Z^{\prime}$. We will denote the non-negative elements of $Z$ by $N$ and the fraction field of $Z$ by $Q$. We start by transferring some of the coding techniques, in particular recursion, to our setting. As most of the proofs are direct transfers of the classical ones, we leave most of the details to the reader.

It is already clear that $N$ is a model of first-order arithmetic. 
Lemma 24. There is a definable map $\beta: N \times N \rightarrow N$ such that for each $l \in N$ and each definable map $f: N_{\leq l} \rightarrow N$ there is $k \in N$ such that $\beta(k, i)=f(i)$ for $i \leq l$.

Proof. Since the function $f$ may definable using parameters outside $N$, we will remind the proof (we refer to [19, $\S I I .2]$ for the details). Since $N$ is a model of first-order arithmetic, there is a definable bijection $\theta: N \times N \rightarrow N$. Define $\beta^{\prime}(r, a, i):=\operatorname{rem}(r,(i+1) \cdot a+1)$, where $\operatorname{rem}(x, y)$ denotes the remainder after integer division of $x$ by $y$. Let $\beta(k, i):=\beta^{\prime}\left(\theta^{-1}(k), i\right)$.

Let $l \in N$ and $f: N_{<l} \rightarrow N$ be definable. It is left to show that there exist $r, a \in N$ such that $\beta^{\prime}(r, a, i)=f(i)$ for $i \leq l$. Since $N$ is a model of first-order arithmetic, we can find $a \in N$ such that $f(i)<a$ for each $i \leq l$ and all elements of

$$
\left\{(i+1) a+1: i \in N_{\leq l}\right\}
$$

are pairwise coprime. We denote $(i+1) a+1$ by $k_{i}$. To finish the construction we just need to establish the following claim.

Claim 1. For each $m \in N_{\leq l}$, there exists $r \in N$ such that for each $i \in N_{\leq m}$

$$
\operatorname{rem}\left(r, k_{i}\right)=f(i) .
$$

Suppose not. Let $m \in N_{\leq l}$ be minimal such that $r$ as in the claim does not exist. By minimality of $m$, there is $r^{\prime} \in N$ be such that for every $i \in \mathbb{N}_{\leq m-1}$

$$
\operatorname{rem}\left(r^{\prime}, k_{i}\right)=f(i) \text {. }
$$

Note that the set $\left\{k_{i}: i \in N_{<m}\right\}$ is definable inside $N$. Let $r \in N$ such that $\operatorname{rem}\left(r, k_{i}\right)=\operatorname{rem}\left(r^{\prime}, k_{i}\right)$ for each $i<m$, and $\operatorname{rem}\left(r, k_{m}\right)=f(m)$. Such an $r$ exists by the Chinese Remainder Theorem in $N$. The Chinese Remainder holds in $N$ because $N$ is a model of first-order arithmetic. Contradiction.

From the proof of the above Lemma, it is clear that $\beta$ is already definable in $\langle N,+, \cdot,<\rangle$, and hence for every $l \in N$, every definable subset of $N_{<l}$ is definable in $\langle N,+, \cdot,<\rangle$.

Lemma 25. Let $c: K^{n} \rightarrow N$ and $g: K^{n} \times N \rightarrow N$ be definable. Then there is a unique definable function $f: K^{n} \times N \rightarrow N$ such that for all $a \in K^{n}$

$$
\begin{aligned}
f(a, 0) & =c(a), \\
f(a, i+1) & =g(a, f(a, i)) .
\end{aligned}
$$

Proof. As in the real case, given $a \in K^{n}$ and $j, l \in N$, we define $f(a, j)=l$ if there exists $k \in N$ such that

$$
\begin{aligned}
\beta(k, 0) & =a ; \\
\beta(k, j) & =l ; \\
\forall i \in N \text { such that } i<j, \quad \beta(k, i+1) & =g(a, \beta(k, i)) .
\end{aligned}
$$

Corollary 26. Let $X \subseteq N$ be unbounded and definable. Then there is definable bijection from $N$ to $X$.

Proof. Let $f: N \rightarrow X$ be the function that takes 0 to the minimum of $X$ and $i+1$ to the successor of $f(i)$ in $X$. By Lemma $25 f$ is definable. 
Definition 27. Let $A$ and $B$ be definable sets. Let $\Delta$ be a family of functions from $B$ to $K^{m}$. We say that $\Delta$ is in definable bijection with $A$ if there exists a definable map $\alpha: A \times B \rightarrow K^{m}$ such that:

(1) for every $f \in \Delta$ there exists a unique $a \in A$ such that for every $b \in K^{n}$ $f(b)=\alpha(a, b)$

(2) for every $a \in A$ the map $x \mapsto \alpha(a, x)$ is in $\Delta$.

With the above notation, we denote by $\widehat{\alpha}: \Delta \rightarrow A$ the map sending $f \in \Delta$ to the unique $a \in A$ satisfying (1).

If $\Gamma$ is a family of subsets of $B$, we say that $\Gamma$ is in definable bijection with $A$ if the family of characteristic functions of the sets in $\Gamma$ is in definable bijection with $A$. By abuse of notation, if $\alpha: A \times B \rightarrow\{0,1\}$ is the corresponding map, we denote by $\widehat{\alpha}: \Gamma \rightarrow A$ the map sending $X \in \Gamma$ to the unique $a \in A$ satisfying the analogue of (1).

Example 28. The family of open balls in $K^{n}$ is in definable bijection with $K^{n} \times$ $K_{>0}$.

Lemma 29. The family of definable bounded subsets of $N$ is in definable bijection with $N$.

Proof. Let $C \subseteq N$ be the set of all $k \in N$ such that $\beta(k, i) \in\{0,1\}$ for all $i \in N$. Define $\gamma: C \times N \times N \rightarrow\{0,1\}$ by

$$
(k, l, i) \mapsto \begin{cases}\beta(k, i), & \text { if } i \leq l ; \\ 0, & \text { otherwise. }\end{cases}
$$

Since $N$ is a model of first-order arithmetic, there is a definable bijection $\theta: N \times$ $N \rightarrow N$. Let $D$ be $\theta(C \times N)$. Now consider the subset $E$ of $D$ containing all $k \in N$ such that there is no $k^{\prime} \in N$ with $k^{\prime}<k$ and

$$
\left\{i \in N: \gamma\left(\theta^{-1}\left(k^{\prime}\right), i\right)=1\right\}=\left\{i \in N: \gamma\left(\theta^{-1}(k), i\right)=1\right\} .
$$

By Lemma 24, for every bounded definable subset $X$ of $N$ there is $k \in D$ such that $\left\{i \in N: \gamma\left(\theta^{-1}(k), i\right)=1\right\}=X$. Hence by Fact 1 there is a unique $k \in E$ with this property. Hence the family of definable bounded subsets of $N$ is in definable bijection with $E$. By Corollary $26 E$ is in definable bijection with $N$. Thus the family of definable bounded subsets of $N$ is in definable bijection with $E$.

Corollary 30. The family of definable subsets of $N$ is in definable bijection with $K$.

We denote by $\widehat{\epsilon}$ the corresponding bijection.

Proof. The idea of the proof is to use the expansion in base 2 to encode definable subsets of $N$ as elements of $K$. Let $E$ be the family of all definable subsets of $N$ and $C$ be the family of unbounded definable subsets of $N$. We want to prove that $E$ is in definable bijection with $K$.

Claim 1. $C$ is in definable bijection with $(0,1]$.

The Corollary then follows: in fact, by Lemma 29, $E \backslash C$ is in definable bijection with $N$. Moreover, the disjoint union of $K$ and $N$ is in definable bijection with $K$ : we define $\mu: K \sqcup N \rightarrow K$ as follows:

$$
\mu(x):= \begin{cases}x & \text { if } x \in K \backslash N \\ 2 x & \text { if } x \text { is in the copy of } N \text { inside } K \\ 2 x+1 & \text { if } x \text { is in the copy of } N \text { outside } K .\end{cases}
$$


Hence, $E$ is in definable bijection with $K$.

Let us prove now Claim 1. By Lemma 25 there is a unique definable function from $N$ to $N$, which we denote by $2^{n}$, such that $2^{0}=1$ and $2^{n+1}=2 \cdot 2^{n}$. For $i \in\{0,1\}$ let

$Y_{n, i}:=\left\{a \in(0,1]:\right.$ there is $m \in 2 \cdot N+i$ such that $\left.m<2^{n} a \leq m+1\right\}$.

Given $X \subset N$ definable and unbounded and $n \in N$, let $f: N \rightarrow\{0,1\}$ be the characteristic function of $X$, and

$$
Z_{n, X}:=\bigcap_{\ell \in N_{\leq n}} Y_{\ell, f(\ell)}
$$

By induction on $n$, it is easy to see that

$$
Z_{n, X}=\left(\frac{a_{n}}{2^{n}}, \frac{a_{n}+1}{2^{n}}\right]
$$

for some unique $a_{n} \in N$ with $0 \leq a_{n}<2^{n}$. Let $a:=\limsup _{n \rightarrow \infty} \frac{a_{n}}{2^{n}}$. Then, since we assumed that $X$ is unbounded, it is easy to see that $\bigcap_{n \in N} Z_{n, X}=\{a\}$ and $a \in(0,1]$. Define $\widehat{\lambda}(X):=a$.

We now show that $\widehat{\lambda}$ is a definable bijection between $C$ and $(0,1]$. Given $a \in(0,1]$ let $X:=\left\{n \in N: a \in Y_{n, 1}\right\}$ : then, $\widehat{\lambda}(X)=a$, and hence $\widehat{\lambda}$ is surjective. Let $X, X^{\prime}$ be distinct definable unbounded subsets of $N$, and assume, for a contradiction, that $b:=\widehat{\lambda}(X)=\widehat{\lambda}\left(X^{\prime}\right)$. Let $n:=\min \left(X \Delta X^{\prime}\right)$; w.l.o.g., we can assume $n \in X \backslash X^{\prime}$. Then, $Z_{n, X^{\prime}}=\left(\frac{a_{n}-1}{2^{n}}, \frac{a_{n}}{2^{n}}\right]$ and $Z_{n, X}=\left(\frac{a_{n}}{2^{n}}, \frac{a_{n}+1}{2^{n}}\right]$ for a unique $a_{n} \in N$ with $1 \leq a_{n}<2^{n}$. Moreover, since $b=\widehat{\lambda}(X)=\widehat{\lambda}\left(X^{\prime}\right)$, we have $a_{n}=b \cdot 2^{n}$, and, for every $m>n, m \in X^{\prime}$ and $m \notin X$; however, the latter contradicts the fact that $X$ is unbounded. $Y_{n, 1}$.

The corresponding function $\lambda: K \times N \rightarrow\{0,1\}$ is given $\lambda(a, n)=1 \leftrightarrow a \in$

Lemma 31. The family of definable functions from $N$ to $K$ is in definable bijection with $K$.

Proof. The idea of the proof is that $K^{N} \approx\left(2^{N}\right)^{N} \approx 2^{N \times N} \approx 2^{N} \approx K$, where $A^{B}$ denotes the family of definable functions from $B$ to $A$, and $A \approx B$ means that there is a definable bijection between $A$ and $B$.

More in details, fix a definable bijection $\theta: N \times N \rightarrow N$. Given $f: K \rightarrow N$ definable, let $X_{f}$ be the definable subset of $N$ such that, for every $i, j \in N$,

$$
j \in \widehat{\epsilon}^{-1}(f(i)) \leftrightarrow \theta(i, j) \in X_{f} .
$$

The definable bijection $\widehat{\delta}$ is given by $\widehat{\delta}(f):=\widehat{\epsilon}\left(X_{f}\right)$. Equivalently, define $\widehat{\delta}(f)$ to be the unique $b \in K$ such that, for every $i, j \in N, \epsilon(f(i), j)=\epsilon(b, \theta(i, j))$.

The corresponding function $\delta$ is defined in the following way: for every $b \in K$ and $i \in N, \delta(b, i)$ is the unique $c \in K$ such that, for every $j \in N, \epsilon(c, j)=$ $\epsilon(b, \theta(i, j))$.

Corollary 32. Let $c: K^{n} \rightarrow K$ and $g: K^{n} \times N \rightarrow K$ be definable. Then there is a unique definable function $f: K^{n} \times N \rightarrow K$ such that for all $a \in K^{n}$

$$
\begin{aligned}
f(a, 0) & =c(a), \\
f(a, i+1) & =g(a, f(a, i)) .
\end{aligned}
$$


Notice that from the proofs of Corollary 30 and Lemma 31 it follows that every definable subset of $N$ and every definable function from $N$ to $K$ are already definable in $\langle K, N,+, \cdot,<\rangle$.

Moreover, we can encode definable subsets of $N$ as elements of $\mathbb{K}$. Thus, the set sort of the proposed model of arithmetic is $\mathbb{K}$ itself, and the inclusion relation $\in$ is defined as follows:

$$
n \in a \leftrightarrow \epsilon(a, n)=1 .
$$

Finally, we add a function, predicate or constant for (the translation via $\widehat{\epsilon}$ of) every function, predicate, or constant in the language of $\mathbb{K}$. It is now clear that the resulting structure is a model of second-order arithmetic.

5.2. From models of arithmetic to unrestrained structures. Conversely, start with $\mathcal{N}:=\langle N, D ; 0,1,+, \cdot,<, \ldots\rangle$ a model of second-order arithmetic, in the sense explained at the beginning of 95 , in the language $L$. Let $Z$ be the ring generated by $N$ and $Q$ be the field of fractions of $Z$. As in [19, Def. I.4.2], a set of "real numbers" can be interpreted inside $\mathcal{N}$ : more precisely, a "sequence of rational numbers" is a definable function from $N$ to $Q$; such a sequence is Cauchy if it satisfies the usual Cauchy condition. We set $K$ to be the set of Cauchy sequences modulo the null sequences, with the operations,$+ \cdot$ and order $<$ induced by the ones on $Q$. Clearly, $Q$ embeds definably and canonically in $K$. It is also clear that $\langle K, \cdot,+,<\rangle$ is an ordered field; moreover, since the family of Cauchy sequences of rational numbers is a definable family, $K$ is interpretable in $\mathcal{N}$.

Lemma 33. $\mathbb{K}_{0}:=\langle K, Z, \cdot,+,<\rangle$ is a definably complete structure.

Proof. Standard proof of analysis (cf. [19, Theorem III.2.2]). Let $A \subset K$ be definable, bounded, and nonempty; we have to show that $A$ has a least upper bound. W.l.o.g., we can assume that $A$ is an initial segment, that is, if $a \in A$ and $b<a$, then $b \in A$; moreover, we can also assume $0 \in A$.

For every $n \in N$, let

$$
f(n):=\max \left\{\frac{m}{2^{n}}: m \in N, \frac{m}{2^{n}} \in A\right\} .
$$

By definition, $f$ takes values in $Q \cap A$; it is clear that $f$ is a Cauchy sequence, and that its equivalence class is the l.u.b. of $A$.

Thus, we have the function $\widehat{\epsilon}$ for the structure $\mathbb{K}_{0}$; using the coding given by $\widehat{\epsilon}$ we can translate all the extra functions, predicates and constants in $L$ as functions, predicates, and constants on $K$; we denote by $\mathbb{K}$ the resulting expansion of $\mathbb{K}_{0}$. It is still true (with the same proof as in Lemma 33) that $\mathbb{K}$ is definably complete, and thus we showed how to transform a model of second-order arithmetic into an unrestrained definably complete structure.

The two transformations are inverse to each other; thus we can say that models of second-order arithmetic and unrestrained definably complete structures are the same objects.

\section{Definable functions and Meager Sets}

In this section we will establish some preliminary facts about definable functions, and show how to transfer part of the theory about Baire category to the definable context. We will later use these facts to prove Theorem B 
6.1. Definably meager and $D_{\Sigma}$ sets. In order to shows how to use Theorem A, and because we will use it in the remainder of this section, we give a quick new proof of a conjecture by Fornasiero and Servi [5]. It was first proved by different methods in 8].

Definition 34. A definable set $A \subseteq K^{n}$ is called definably meager if $A=$ $\bigcup_{t \in K} X_{t}$, for some definable increasing family $\left(X_{t}: t \in K\right)$ of nowhere dense subsets of $K^{n}$.

Lemma 35. Let $A \subseteq K^{n}$ be at most pseudo-enumerable. Then $A$ is definably meager.

Proof. Since $A$ is at most pseudo-enumerable, there exists a definable closed and discrete set $D \subset K_{\geq 0}$ and a definable surjective function $f: D \rightarrow A$. For each $t \in K$, let $X_{t}:=f\left(D_{\leq t}\right)$. By Fact 7 each $X_{t}$ is pseudo-finite. Then $A=\bigcup_{t \in K} X_{t}$, and $\left(X_{t}: t \in K\right)$ is definable increasing family of nowhere dense subsets of $K^{n}$.

Theorem 36 ([8]). $K$ is not definably meager.

Proof. If $\mathbb{K}$ is restrained, then Theorem A and [3] Proposition 6.4] shows that every definably meager sets has empty interior, and in particular $K$ is not definably meager.

If not, then, as shown in [3. Lemma 6.2], we can mimic one of the classical proofs of Baire's category theorem to conclude that $K$ is not definably meager.

Definition 37. Let $X \subseteq K^{n}$ be a definable set. We say that $X$ is a $D_{\Sigma}$ set if it is the union of a definable increasing family, indexed by $K$, of closed subsets of $K^{n}$.

By [5. Remark 3.3], a definable set is a $D_{\Sigma}$ set iff it is the projection of a definable closed set.

Lemma 38. Let $A \subseteq K^{n+m}$ be a $D_{\Sigma}$ set. Let

$$
T(A):=\left\{x \in K^{n}: A_{x} \text { is definably meager }\right\} .
$$

Then $A$ is definably meager iff $K^{n} \backslash T(A)$ is definably meager.

Proof. It follows immediately from [5, Lemma 5.2 and Proposition 5.4].

6.2. Definable functions and continuity. Now that we have a reasonable analogue of the notion of meager sets, we can use it to transfer several well-known results from real analysis to $\mathbb{K}$. For the remainder of this subsection we will not use Theorem A anymore. Afterwards, we will use these results to prove Theorem B by distinguishing the case when $\mathbb{K}$ is either restrained or unrestrained.

First, we show that a monotone function $f$ is continuous outside a "small" set.

Lemma 39. Let $f: K \rightarrow K$ be a definable monotone function. Then, the set $\mathcal{D} f$ of discontinuity points of $f$ is at most pseudo-enumerable.

Proof. For every $\varepsilon>0$ let

$$
\begin{aligned}
\mathcal{D} f(\varepsilon) & :=\left\{x \in K: \limsup _{y \rightarrow x}|f(y)-f(x)|>\varepsilon\right\} \\
& =\left\{x \in K: \lim _{y \rightarrow x^{+}} f(y)-\lim _{y \rightarrow x^{-}} f(y)>\varepsilon\right\} .
\end{aligned}
$$

It is easy to see that $f(\mathcal{D} f(\varepsilon))$ is discrete for every $\varepsilon>0$. Thus, by Fact 10 , since $\mathcal{D} f=\bigcup_{\varepsilon>0} \mathcal{D} f(\varepsilon), \mathcal{D} f$ is at most pseudo-enumerable. 
Definition 40. Let $f: K \rightarrow K$ be a definable function. The four Dini derivatives of $f$ are:

$$
\begin{aligned}
\lambda_{\ell} f(x) & :=\liminf _{y \rightarrow x^{-}} \frac{f(y)-f(x)}{y-x} \\
\lambda_{r} f(x) & :=\liminf _{y \rightarrow x^{+}} \frac{f(y)-f(x)}{y-x} \\
\Lambda_{\ell} f(x) & :=\limsup _{y \rightarrow x^{-}} \frac{f(y)-f(x)}{y-x} \\
\Lambda_{r} f(x) & :=\limsup _{y \rightarrow x^{+}} \frac{f(y)-f(x)}{y-x} .
\end{aligned}
$$

Lemma 41. Let $f: K \rightarrow K$ be definable and continuous. If, for every $x \in K$, $\Lambda_{r} f(x) \in K$ and $\Lambda_{r} f$ is continuous, then $f$ is $\mathcal{C}^{1}$ (and $f^{\prime}=\Lambda_{r} f$ ).

Proof. As in [1, Theorem 1.3].

We will now adapt the classical definition of Baire class to the "definable" context.

Definition 42. Let $X \subseteq K^{n}$ be a definable set, $f: X \rightarrow K$ be a definable function, and $n \in \mathbb{N}$. We say that $f$ is of definable Baire class $n$ if:

(1) either $n=0$ and $f$ is continuous;

(2) or $n>0$ and there exists a definable family of functions $\left(f_{t}: X \rightarrow K\right)_{t \in K}$ such that each $f_{t}$ is of class $(n-1)$ and

(a) either, for every $x \in X, f(x)=\lim _{t \rightarrow+\infty} f_{t}(x)$.

(b) or, for every $x \in X, f(x)=\sup _{t} f_{t}(x)$;

(c) or, for every $x \in X, f(x)=\inf _{t} f_{t}(x)$.

In the above definition we had to add Clauses (2-b) and (2-c) to the classical definition, because we could not prove that a function satisfying e.g. Clause (2-b) would satisfy Clause (2-a).

The interest for us of the above definition stems from the following fact.

Lemma 43. Let $f: K \rightarrow K$ be definable and continuous. Then, $\Lambda_{r} f$ is of definable Baire class 2.

Proof. For every $t \neq 0$ let $g_{t}(x):=\frac{f(x+t)-f(x)}{t}$. Then,

$$
\Lambda_{r} f(x)=\inf _{t>0} \sup _{0<s<t} g_{s}(x) .
$$

Definition 44. Let $f: X \rightarrow K$ be a definable function. We say that $f$ is almost continuous if the set of its discontinuity points $\mathcal{D} f$ is nowhere dense.

Definition 45. $\mathbb{K}$ has locally o-minimal open core if there does not exist a definable, closed, discrete, and unbounded subset of $K_{\geq 0}$ (see [4, Thm. 3.3]).

We could prove the following lemma only under the assumption that $\mathbb{K}$ does not have locally o-minimal open core.

Lemma 46. Assume that $\mathbb{K}$ does not have locally o-minimal open core. Let $\left(f_{t}\right.$ : $\left.K^{n} \rightarrow[0,1]\right)_{t \in K}$ be a definable family of almost continuous functions. Let $f$ : $K^{n} \rightarrow[0,1]$ be either of the following functions:

(1) $f(x)=\sup _{t} f_{t}(x)$; 
(2) or $f(x)=\lim _{t \rightarrow \infty} f_{t}(x)$.

Then, the restriction of $f$ to the complement of a definably meager set is continuous. If moreover each $f_{t}$ is continuous (i.e., $f$ is of definable Baire class 1 ), then $\mathcal{D}(f)$ is definably meager.

Proof. Minor variation of [15, Thm. 7.3]. Let $M \subset K_{\geq 0}$ be definable, closed, discrete, and unbounded.

Let $D_{i}$ be the closure of $\mathcal{D} f_{i}$, and $D:=\bigcup_{i \in M} D_{i}$ since each $D_{i}$ is nowhere dense, $D$ is definably meager. Let $X:=K^{n} \backslash D$ : notice that $X$ is dense in $K^{n}$. We claim that $f \Gamma_{X}$ is continuous outside a definably meager set. (If each $f_{i}$ is continuous, then $D$ is empty, and we also obtain the "moreover" clause).

For every $\varepsilon>0$, set

$$
F_{\varepsilon}:=\{a \in X: \forall \delta>0 \exists x \in X(|x-a|<\delta \&|f(x)-f(a)|>5 \varepsilon)\} .
$$

it suffices to show that $F_{\varepsilon}$ is nowhere dense. Fix an open box $V \subseteq K^{n}$ and $\varepsilon>0$.

We prove first Case (2). Notice that $f(x)=\lim _{t \rightarrow \infty, t \in M} f_{t}(x)$. For every $i \in K$, let

$$
E_{i}:=\left\{x \in V:\left|f_{i}(x)-f(x)\right| \leq \varepsilon\right\} .
$$

Notice that $\left(E_{i}: i \in M\right)$ is a definable family of subsets of $V$, and $\bigcup_{i \in M} E_{i}=V$. Hence, by Theorem [36] there exists $i_{0} \in M$ such that the closure of $E_{i_{0}}$ has nonempty interior. Let $U \subseteq \operatorname{cl}\left(E_{i_{0}}\right)$ be a nonempty open box. Since $f_{i_{0}}$ is continuous on $U \cap X$, after shrinking $U$ we can also assume that, for every $x, x^{\prime} \in U \cap X$, $\left|f_{i_{0}}(x)-f_{i_{0}}\left(x^{\prime}\right)\right| \leq \varepsilon$. Thus, for every $x, x^{\prime} \in U \cap X,\left|f(x)-f\left(x^{\prime}\right)\right| \leq 3 \varepsilon$, and therefore $U \cap X \cap F_{\varepsilon}=\emptyset$.

Thus, every nonempty open definable set $V$ contains a nonempty open set $U$ disjoint from $F_{\varepsilon} \cap X$, and therefore $F_{\varepsilon} \cap X$ is nowhere dense.

The proof of Case (1) is similar, using instead

$$
E_{i}:=\left\{x \in V: f(x) \leq f_{i}(x)+\varepsilon\right\} .
$$

Example 47. (1) Notice that in the above lemma we cannot conclude that $\mathcal{D} f$ is definably meager without also assuming that either each $f_{i}$ is continuous or $\mathbb{K}$ is restrained (see Corollary 501). In fact, it is easy to see that the characteristic function of an at most pseudo-enumerable set is the pointwise limit of a definable family of functions $f_{i}$ such that each $\mathcal{D}\left(f_{i}\right)$ is pseudo-finite. For instance, let $\mathcal{R}:=\langle\mathbb{R},+, \cdot,<, \mathbb{N}\rangle$. Let $f: \mathbb{R} \rightarrow \mathbb{R}$ be the characteristic function of $\mathbb{Q}$ : then, $f$ can be written as the limit of a definable family of functions $f_{i}$, with $\mathcal{D}\left(f_{i}\right)$ finite for every $i$.

(2) Let $f: K^{n} \rightarrow K$ be a definable continuous function such that $\Lambda_{r} f$ is discontinuous on a nonmeager set: then $\Lambda_{r} f$ is a function of definable Baire class exactly 2 (i.e., not 1 ). It is an easy exercise to find such a function $f$ when $\mathbb{K}$ is unrestrained (cf. 11, p. 42]): however, we will see later that when $\mathbb{K}$ is restrained such $f$ does not exist.

(3) If $X \subseteq K^{n}$ is a nonempty definable closed subset, then the characteristic function of $X$ is of definable Baire class 1 .

(4) If $\mathcal{R}$ is an unrestrained expansion of the real field, then, for each $n \in \mathbb{N}$, the "definable Baire class $n$ " and the "Baire class $n$ " are the same class (because all sets in the projective hierarchy are definable in $\mathcal{R}$ ); therefore, by a theorem by Lebesgue [11, for each $n$ there is a definable function of definable Baire class exactly $n$. 
(5) Let $C \subset \mathbb{R}$ be a "Cantor set", i.e., a nonempty, definable, closed, perfect, nowhere dense subset. Define $f: \mathbb{R} \rightarrow \mathbb{R}$ as $f(x)=0$ outside $C, f(x)=1 / 2$ on each point of $C$ such that there exists $\varepsilon>0$ with either $(x, x+\varepsilon) \cap C=\emptyset$, or $(x-\varepsilon, x) \cap C=\emptyset$, and $f(x)=1$ otherwise. Then, $f$ is of definable Baire class exactly 2 (cf. [15, Ch.7]). There are some restrained expansions of $\mathbb{R}$ defining a Cantor set as above. We leave open the question if in the restrained case there can be definable functions of definable Baire class greater than 2 .

(6) If $\mathbb{K}$ is restrained and defines set $X \subset K$ which is dense and codense, then the characteristic function of $X$ is not in any definable Baire class; for instance, if $\mathcal{R}$ is the expansion of the real field by the set $R^{\text {alg }}$ of real algebraic numbers, then the characteristic function of $R^{a l g}$ is of Baire class 2, but it is not in any definable Baire class.

6.3. Restrained structures. In this subsection we will prove a few results about definable functions and sets in restrained structures. We will use them to prove the restrained case of Theorem B however, we think that some of them are of independent interest.

Lemma 48. Let $X \subseteq K$ be definable and nowhere dense. Then, there exists two sets $Y, Z \subset K$ discrete, definable, and such that $Y \subseteq X$ and $\operatorname{cl}(X) \subseteq \operatorname{cl}(Y) \cup \operatorname{cl}(Z)$. Moreover, the choice of $Y$ can be made in a uniform way: that is, if $X \subset K^{n+1}$ is definable, and for every $t \in K^{n}, X_{t}$ is nowhere dense, then there exists $Y, Z \subset K^{n+1}$ definable, such that $Y \subseteq X$ and, for every $t \in K^{n}, Y_{t}$ and $Z_{t}$ are discrete, and $X_{t} \subseteq \operatorname{cl}\left(Y_{t}\right) \cup \operatorname{cl}\left(Z_{t}\right)$.

Proof. Let $Y$ be the set of isolated points of $X$. W.l.o.g., we can assume that $X$ is closed and $X \subset(0,1)$. Thus, $(0,1) \backslash X$ can be written in a unique way as a union of disjoint open intervals; let $Z$ be the set of centers of such intervals.

Lemma 49 . $\mathbb{K}$ is restrained iff, for every $m \in \mathbb{N}$, every definably meager subset of $K^{m}$ is nowhere dense.

Proof. For the "if" direction, let $X \subset K$ be at most pseudo-enumerable. Then, by Lemma 35 is definably meager; thus, by assumption, $X$ is nowhere dense, proving that $\mathbb{K}$ is restrained.

For the "only if" direction, first we assume $m=1$. If $\mathbb{K}$ has locally o-minimal open core, then the conclusion holds (see [4, Theorem 3.3]). Otherwise, there exists an unbounded definable closed discrete set $D \subset K_{\geq 0}$. Let $X \subset K$ be definably meager; thus, $X=\bigcup_{i \in K} Y_{i}$, for some $\left(Y_{i}: i \in K\right)$ definable increasing family of nowhere dense set. Since $D$ is unbounded, $X=\bigcup_{i \in D} Y_{i}$. By Lemma 48, there exists two definable families of discrete sets $\left(Z_{i}: i \in D\right)$ and $\left(W_{i}: i \in D\right)$, such that, for every $i \in D, Y_{i} \subseteq \operatorname{cl}\left(Z_{i} \cup W_{i}\right)$. Let $T:=\bigcup_{i \in D} Z_{i} \cup W_{i}$. By Fact 10, $T$ is at most pseudo-enumerable, and hence nowhere dense, since $\mathbb{K}$ is restrained. Since $X \subseteq \operatorname{cl}(T)$, we have that $X$ is nowhere dense.

Assume now that $m \geq 1$ (and $\mathbb{K}$ is restrained). By induction on $n$, we show the following:

$(1)_{n}$ Every $D_{\Sigma}$ subset of $K^{n}$ has interior or is nowhere dense;

$(2)_{n}$ For every $p \in \mathbb{N}$ and $A D_{\Sigma}$ subset of $K^{n+p}$, the set $\left\{x \in K^{n}: \operatorname{cl}\left(A_{x}\right) \neq \operatorname{cl}(A)_{x}\right\}$ is definably meager in $K^{n}$.

$(3)_{n}$ If $A$ is a $D_{\Sigma}$ subset of $K^{n}$, then $\operatorname{fr}(A):=\operatorname{cl}(A) \backslash \AA$ is nowhere dense. 
$(4)_{n}$ Every definably meager subset of $K^{n}$ is nowhere dense.

Assertion $(4)_{m}$ is the conclusion of the Lemma. Assertion $(1)_{1}$ is the Case $m=1$.

The proofs of $(2)_{1}$ and the inductive step are as in [14, 1.6].

More precisely, assume that we have already proved $(1)_{n}$; we claim that $(2)_{n}$, $(3)_{n}$, and $(4)_{n}$ also hold. For $(3)_{n}$ : we have

$$
\operatorname{fr}(A)=\operatorname{fr}(\AA) \cup \operatorname{fr}(A \backslash \AA)=\operatorname{fr}(\AA) \cup \operatorname{cl}(A \backslash \AA)
$$

and each of the two pieces is a $D_{\Sigma}$ set with empty interior, and thus, by $(1)_{n}$, nowhere dense.

For $(4)_{n}$, let $X \subseteq K^{n}$ be definably meager: that is, $X=\bigcup_{t \in K} Y_{t}$, where $\left(Y_{t}\right.$ : $t \in K)$ is a definable increasing family of nowhere dense subsets of $K^{n}$. For each $t \in K$, let $Z_{t}$ be the closure of $Y_{t}$ (inside $\left.K^{n}\right)$; define $W:=\bigcup_{t} Z_{t}$. Then, $W$ is definably meager and hence, by Theorem 36 , with empty interior; moreover, $W$ is a $D_{\Sigma}$ set. Thus, by $(1)_{n}, W$ is nowhere dense, and, since $X \subseteq W, X$ is also nowhere dense.

The proof of $(2)_{n}$ is a bit more involved. Let $A$ be a $D_{\Sigma}$ subset of $K^{n+p}$ and $B:=\left\{x \in K^{n}: \exists y \in \operatorname{cl}(A)_{x} \backslash \operatorname{cl}\left(A_{x}\right)\right\}$. We want to show that $B$ is definably meager.

For each open box $U \subseteq K^{p}$, let $C_{U}:=\left\{\langle x, y\rangle \in \operatorname{cl}(A): y \in U \& \operatorname{cl}\left(A_{x}\right) \cap U=\emptyset\right\}$ and $B_{U}:=\pi\left(C_{U}\right)$, where $\pi: K^{n+p} \rightarrow K^{n}$ is the projection onto the first $n$ coordinates. Notice that $B$ is the union of all the $B_{U}$ 's.

Claim 1. For each open box $U, B_{U}$ is nowhere dense.

In fact, let $G:=\pi\left(A \cap\left(K^{n} \times U\right)\right)$. Then, $G$ is a $D_{\Sigma}$ set, and $\operatorname{fr}(G)$ has empty interior (by $\left.(3)_{n}\right)$. However, $B_{U} \subseteq \operatorname{fr}(G)$, and the claim is proved.

For each $r>0$, let

$$
D(r):=\left\{\langle x, y\rangle \in \operatorname{cl}(A):|y| \leq r \& d\left(y, A_{x}\right) \geq r\right\},
$$

$E(r):=\operatorname{cl}(D(r))$, and $F(r):=\pi(E(r))$. Since $B=\bigcup_{r>0} \pi(D(r)) \subseteq \bigcup_{r>0} F(r)$, and each $F(r)$ is closed, it suffices to show that each $F(r)$ has empty interior. Assume, for a contradiction, that $F(r)$ contains a nonempty open box $V$, for some $r>0$. Define $f: V \rightarrow K^{p}, f(x):=\operatorname{lex} \min \left(E(r)_{x}\right)$. By [2, 2.8(1)], the set of discontinuity points of $f$ is definably meager; thus, by $(1)_{n}$, after shrinking $V$ if necessary, we can assume that $f$ is continuous on $V$. Thus, $\Gamma(f)$, the graph of $f$, is contained in $E(r)$. After shrinking $V$ if necessary, by continuity of $f$, we can find an open box $U \subset K^{p}$ of diameter less than $r$ and such that $f(V) \subseteq U$.

Then, $D(r)_{U}:=D(r) \cap\left(K^{n} \times U\right) \subseteq C_{U}$, and therefore

$$
V \subseteq \pi\left(\operatorname{cl}\left(D(r)_{U}\right)\right) \subseteq \operatorname{cl}\left(\pi\left(D(r)_{U}\right)\right) \subseteq \operatorname{cl}\left(B_{U}\right),
$$

contradicting Claim 1 .

Finally, assume that we have already proved all the statements for every $n^{\prime}<n$; we want to prove $(1)_{n}$. Let $A \subset K^{n}$ be a $D_{\Sigma}$ set with empty interior; we want to prove that $A$ is nowhere dense. Notice that $A$ is definably meager; thus, by Lemma 38. the set of points $x \in K^{n-1}$ such that $A_{x}$ has nonempty interior is definably meager; hence, by $(1)_{1}$ and $(4)_{n-1}$, the set of points $x \in K^{n-1}$ such that $A_{x}$ is somewhere dense is nowhere dense. By $(2)_{n-1}$, the set of points $x \in K^{n-1}$ such that $\operatorname{cl}(A)_{x}$ has interior is nowhere dense. Hence, $\operatorname{cl}(A)$ has empty interior.

Corollary 50. Let $\mathbb{K}$ be restrained and without locally o-minimal open core, $n, m \in$ $\mathbb{N}$, and $f: K^{m} \rightarrow K$ be of definable Baire class $n$. Then, $f$ is almost continuous. 
Proof. By induction on $n$, Lemma 46, and Lemma 49.

Lemma 51. Let $\mathbb{K}$ be restrained, $U \subseteq K^{n}$ be open and definable, $f: U \rightarrow K$ be a definable continuous function, and $p \in \mathbb{N}$. Then, $f$ is $\mathcal{C}^{p}$ on a dense open subset of $U$.

Proof. Let $B \subseteq U$ be an open box; it suffices to prove the result for $f \uparrow_{B}$; since $B$ is diffeomorphic to $K^{n}$ itself, it suffices to treat the case when $U=K^{n}$.

If $\mathbb{K}$ has locally o-minimal open core, then, since $f$ is definable in the open core of $\mathbb{K}$, the conclusion follows from [4, Theorem 5.11].

Otherwise, by induction, it suffices to treat the case $p=1$. First, we do the case $n=1$. By Lemma 43, $\Lambda_{r} f$ is of definably Baire class 2. By Corollary 50, $\Lambda_{r} f: K \rightarrow K \cup\{ \pm \infty\}$ is continuous on a dense open set $U$, but may take value infinity somewhere.

Claim 1. Let $V:=\left\{x \in U: \Lambda_{r} f(x) \in K\right\}$. Then, $V$ is open and dense.

If not, since $\Lambda_{r} f$ is continuous on $U$, there would exist an interval $[a, b] \subseteq U$ such that

(1) either for every $x \in[a, b], \Lambda_{r} f(x)=+\infty$,

(2) or, for every $x \in[a, b], \Lambda_{r} f(x)=-\infty$.

By replacing $f(x)$ with $f(x)-\frac{f(b)-f(a)}{b-a}(x-a)$, w.l.o.g. we can assume that $f(b)=$ $f(a)$. Thus, since $f$ is continuous and definable, there exists $x_{0} \in(a, b)$ that is a maximum for $f$ in $[a, b]$; but then $\Lambda_{r} f\left(x_{0}\right) \leq 0$, contradicting Case (1). Similarly, there exists $x_{1} \in(a, b)$ that is a minimum for $f$ in $(a, b)$, contradicting Case (2). Finally, by Lemma 41$]$ is $\mathcal{C}^{1}$ on $V$.

Assume now that $n>1$. We will prove that, outside some nowhere dense set, each partial derivative of $f$ exists and is continuous; it suffices to show that $\partial f / \partial x_{n}$ exists and is continuous on a dense open set. Let $\bar{e}_{n}:=\langle 0, \ldots, 0,1\rangle \in K^{n}$. Define the Dini derivatives of $f$ in the direction $\bar{e}_{n}$ as $\Lambda_{r} f:=\lim _{\sup _{t \rightarrow 0^{+}}} \frac{f\left(x+t \bar{e}_{n}\right)-f(x)}{t}$, and similarly for the other three Dini derivatives. Reasoning as in the case $n=1$, we see that $\Lambda_{r} f$ is finite and continuous on a dense open set $U$, and similarly for the other three Dini derivatives. It then suffices to show that, after maybe shrinking $U$ to a smaller dense open definable set, the four Dini derivatives coincide; by symmetry, it suffices to prove that $\lambda_{\ell} f=\Lambda_{r} f$ on a dense open set. Assume not: then, by continuity, there would exists an open set $V$ such that $\lambda_{\ell} f(x) \neq \Lambda_{r} f(x)$ for every $x \in V$; but this contradicts the case $n=1$.

\section{LeBesGue's TheOREM}

We give now an application of Theorem A, by proving the following analogue of Lebesgue's theorem. Remember that we call $\mathbb{K}$ unrestrained if it defines a discrete subring (with 1), and restrained otherwise.

Theorem B. Let $f: K \rightarrow K$ be a definable monotone function. Then, $f^{\prime}(x)$ exists and is in $K$ (i.e., not $\pm \infty$ ) on a dense subset of $K$.

The reasons we chose this example are that it is interesting in its own right (it was conjectured in [12]), and it gives a good illustration of how Theorem A can be used to transfer various classical results from $\mathbb{R}$ to $\mathbb{K}$. Theorem A allows us to reduce the proof of the above Theorem to structures satisfying either condition (I) or (II) of Theorem A. 
7.1. The restrained case. We will now give a proof of Theorem $B$ in the case when $\mathbb{K}$ is restrained.

The theorem in the restrained case follows immediately from the results in $\lcm{6.3}$ plus the following lemma.

Lemma 52. Let $\mathbb{K}$ be restrained; let $f: K \rightarrow K$ be a definable monotone function. Then, there exists a definable closed nowhere dense set $C$ such that $f$ is continuous outside $C$.

Proof. Let $D$ be the set of discontinuity points of $f$, and $C$ be its closure. By Lemma 39, $D$ is at most pseudo-enumerable; by Theorem A, $C$ is nowhere dense.

The following corollary concludes the proof of Theorem B in the case when $\mathbb{K}$ is restrained.

Corollary 53. Let $\mathbb{K}$ be restrained; let $f: K \rightarrow K$ be a definable monotone function. Then, $f$ is $\mathcal{C}^{1}$ outside a nowhere dense set.

Proof. By Lemmas 52 and 51

7.2. Measure theory. Let us examine now the case when $\mathbb{K}$ defines a discrete subring $Z$. Using the results in $\$ 5$, we can transfer the tools of measure theory. We will sketch the relevant ideas in the following (cf. [19, §X.1] for a different approach). Many of the definitions make sense also in the case when $\mathbb{K}$ is restrained: therefore in this subsection, unless said otherwise, we are not assuming that $\mathbb{K}$ is unrestrained.

Definition 54. Let $D \subset K_{\geq 0}$ be a nonempty closed discrete definable set, and let $s_{D}$ be defined as in Definition 3 Let $h: D \rightarrow K$ be a definable function. We define $H: D \rightarrow K$ to be function given recursively by $H(\min (D))=0$ and for every $d \in D$ with $d \neq \max (D), H\left(s_{D}(d)\right)=H(d)+h(d)$. If $h$ takes only nonnegative values and $H$ exists, we denote

$$
\sum_{d \in D} h(d):=\sup _{d \in D} H(d) \in K_{\geq 0} \cup\{+\infty\} .
$$

It is easy to see that if $H$ is definable, then it is unique. Moreover, if $\mathbb{K}$ is unrestrained, then $H$ exists by Corollary 32

Definition 55 (Lebesgue measure). Let $a<b \in K \cup\{ \pm \infty\}$; we set $|(a, b)|:=b-a$. Let $\mathcal{U}:=\left(I_{d}: d \in D\right)$ be a definable family of open intervals, indexed by a closed discrete set $D \subseteq K_{\geq} 0$. We define $M(\mathcal{U}):=\sum_{d \in D}\left|I_{d}\right|$ (if it exists).

Let $A \subseteq K$ be a definable set. We denote by $\mu(A)$ the infimum of $M(\mathcal{U})$, as $\mathcal{U}$ varies among all the definable coverings of $A$ by open intervals, indexed by some definable discrete subset of $K_{>0}$, such that $M(\mathcal{U})$ exists. Notice that $\mu(A)$ may not lie in $K$ (since it is the infimum of a set that may not be definable), but in the Dedekind-MacNeille completion of $\langle K,<\rangle$. Notice also that $0 \leq \mu((0,1)) \leq 1$.

Notice that when $\mathbb{K}$ expands $\langle\mathbb{R},+, \cdot, \mathbb{N}\rangle$, then $\mu(X)$ is the outer Lebesgue measure of $X$.

Conjecture 56. $\mu((0,1))=1$.

However, things are much simpler if $\mathbb{K}$ unrestrained. In that case, $M(\mathcal{U})$ always exists, and we can always assume that the index set of $\mathcal{U}$ is either $N$ or an initial 
segment of $N$ (more precisely, for every definable closed discrete subset $D \subset K_{\geq 0}$ there is a unique definable increasing bijection between a unique initial segment of $N$ and $D)$.

Moreover, the family of definable covers of a given definable set $A$ by open intervals indexed by $N$ is itself definable (by Lemma 31), and therefore $\mu(A) \in$ $K_{\geq 0} \cup\{+\infty\}$. Moreover, again by using Lemma 31, if $\left(A_{i}: i \in I\right)$ is a definable family, then $f: i \mapsto \mu\left(A_{i}\right)$ is a definable function.

Proposition 57. Let $\mathbb{K}$ be unrestrained. Then, $\mu((0,1))=1$.

The proof of the above proposition is a minor modification the classical one that $(0,1)$ has Lebesgue measure 1 , and is left to the reader; he can base it on the following result, whose proof is also left to the reader.

Lemma 58 (Commutativity of addition). Let $\mathbb{K}$ be unrestrained. Let $h: N \rightarrow$ $K_{>0}$ be a definable function, and $\sigma: N \rightarrow N$ be a definable bijection. Then, $\sum_{d \in N} h(d)=\sum_{d \in N} h(\sigma(d))$.

Notice that we are not able to prove the above lemma without the assumption that $\mathbb{K}$ defines a discrete subring.

Conjecture 59. Let $D \subseteq K_{>0}$ be a definable closed discrete subset; let $h: D \rightarrow$ $K_{\geq 0}$ be a definable function, and $\sigma: D \rightarrow D$ be a definable bijection. Then, $\sum_{d \in D} h(d)=\sum_{d \in D} h(\sigma(d))$ (i.e., if the sum on the left exists, then also the one on the right exists and is equal to it).

Lemma 60 (Sigma-subadditivity of measure). Let $\mathbb{K}$ be unrestrained. Let $\left(A_{i}\right.$ : $i \in N)$ be a definable family of subsets of $K$. Then,

$$
\mu\left(\bigcup_{i} A_{i}\right) \leq \sum_{i} \mu\left(A_{i}\right)
$$

In particular, if $\mu\left(A_{i}\right)=0$ for every $i \in N$, then $\mu\left(\bigcup_{i} A_{i}\right)=0$. Therefore, if $A \subset K$ is at most pseudo-enumerable, then $\mu(A)=0$.

Proof. Left to the reader.

Corollary 61. Let $\mathbb{K}$ be unrestrained. Let $X \subseteq K$ be a definable set, and $0 \leq \delta<$ $1 \in K$. Assume that for every interval $I$ we have $\mu(X \cap I) \leq \delta|I|$. Then, $\mu(X)=0$.

Proof. Assume not: let $\mu(X)=c>0$. Fix $0<\varepsilon \in K$ small enough (how small will be clear later). Let $\mathcal{U}:=\left(I_{d}: d \in N\right)$ be a definable family of intervals, such that $M(\mathcal{U})<(1+\varepsilon) c$ and $X \subseteq \bigcup_{d} I_{d}$. Thus, by our assumption on $X$,

$$
\mu(X) \leq \sum_{d} \mu\left(I_{d} \cap X\right) \leq \sum_{d} \delta\left|I_{d}\right| \leq \delta(1+\varepsilon) c .
$$

If we take $\varepsilon$ small enough, we have $\delta(1+\varepsilon)<1$, absurd.

7.3. The unrestrained case. With those tools at our disposal, we can now mimic some of the proofs of Lebesgue's theorem: we will follow the trace of [17] for the case when $f$ is continuous, and of [18] for the general cases.

First, a technical lemma, which is easy to prove for every $\mathbb{K}$, without using Theorem A: the proof is left to the reader (cf. [17] for the details). 
Lemma 62 (Riesz's Rising Sun Lemma). Let $a<b \in K$ and $g:[a, b] \rightarrow K$ be a definable bounded function. For every $x \in[a, b]$, denote

$$
G(x):=\max \left(g(x), \limsup _{y \rightarrow x} g(y)\right) .
$$

Let

$$
E:=\{x \in(a, b):(\exists y \in(x, b]) g(y)>G(x)\} .
$$

Then, $E$ is an open definable subset of $(a, b)$. Moreover, let $\left(a^{\prime}, b^{\prime}\right)$ be a maximal open subinterval of $E$. Then, $\lim \sup _{y \rightarrow a^{\prime}} g(y) \leq G\left(b^{\prime}\right)$.

Lemma 63. Let $\mathbb{K}$ be unrestrained. Let $a<b \in K$, and $f:(a, b) \rightarrow K$ be a definable increasing function. Define

$$
A_{\infty}:=\left\{x \in(a, b): \Lambda_{r} f(x)=+\infty\right\} .
$$

Then, $\mu\left(A_{\infty}\right)=0$.

Proof. The same as in [17, Assertion 1].

More in details, given $c \in K$, define

$$
\begin{aligned}
g(x) & :=f(x)-c x ; \\
A_{c} & :=\left\{x \in(a, b): \Lambda_{r} f(x)>c\right\} ; \\
E_{c} & :=\left\{x \in(a, b):(\exists y>x) g(y)>g\left(x^{+}\right)\right\} ; \\
\mathcal{D} f & :=\{x \in(a, b): f \text { is discontinuous at } x\} .
\end{aligned}
$$

Notice that $\bigcap_{c} A_{c}=A_{\infty}$ and $\mu(\mathcal{D} f)=0$ (because $\mathcal{D} f$ is at most pseudo-enumerable), and therefore it suffices to show that $\mu\left(A_{c} \backslash \mathcal{D} f\right)$ is arbitrarily small for $c$ large enough. Moreover, $A_{c} \backslash \mathcal{D} f \subseteq E_{c}$; therefore, it suffices to show that $\mu\left(E_{c}\right)$ is small.

Let $G$ be as in Lemma 62 notice that $G(x)=g\left(x^{+}\right)$, unless $x=b$, when $G(b)=g(b)$. Thus, by Lemma 62, $E_{c}$ is an open subset of $(a, b)$, and it is the disjoint union of a definable family of open intervals $\left\{\left(a_{k}, b_{k}\right): k \in N\right\}$, such that $c\left(b_{k}-a_{k}\right) \leq f\left(b_{k}^{+}\right)-f\left(a_{k}^{+}\right)$. Hence, $c \sum_{k \in N}\left(b_{k}-a_{k}\right) \leq f(b)-f(a)$, and therefore $\mu\left(E_{c}\right) \leq \frac{f(b)-f(a)}{c}$.

Lemma 64. Let $\mathbb{K}$ be unrestrained. Let $f: K \rightarrow K$ be a definable monotone continuous function.

(1) Let $A:=\left\{x \in K: \lambda_{\ell} f(x)<\Lambda_{r} f(x)\right\}$. Then, $\mu(A)=0$.

(2) The set of points $x \in(a, b)$ such that $f^{\prime}(x)$ does not exist or is infinite has measure 0 .

Proof. We proceed as in [17, Assertion 2]. (2) follows easily from (1), thus we only need to prove (1).

It suffices to show that, for every $0<c<C \in K$, the set

$$
B:=\left\{x \in K: \lambda_{\ell} f(x)<c \& \Lambda_{r} f(x)>C\right\}
$$

has measure 0 . Let $\delta:=c / C$ : by Corollary 61 it suffices to show that, for every $a<b \in K, \mu(B \cap(a, b))<\delta(b-a)$. As in[17, Assertion 2], by applying Lemma62 to the function $g(x):=f(-x)+c x$, we get that $\left\{x \in(a, b): \lambda_{\ell} f(x)<c\right\}$ is contained in an open definable set $D$, such that for every maximal interval $\left(a_{k}, b_{k}\right) \subseteq D$, we have $f\left(b_{k}\right)-f\left(a_{k}\right) \leq c\left(b_{k}-a_{k}\right)$ (notice that we can take the indexes $k$ in $N$ in a definable way). We then apply again Lemma 62 to the function $g(x):=f(x)-C x$ 
restricted to each interval $\left(b_{k}, a_{k}\right)$, and we get that $D \cap\left(b_{k}, a_{k}\right)$ is contained in an open definable set $D_{k}$, such that $\mu\left(D_{k}\right) \leq \frac{f\left(b_{k}\right)-f\left(a_{k}\right)}{C}$. Thus,

$$
\mu(B \cap(a, b)) \leq \sum_{k} \mu\left(D_{k}\right) \leq \frac{\sum_{k} f\left(b_{k}\right)-f\left(a_{k}\right)}{C} \leq \delta \sum_{k}\left(b_{k}-a_{k}\right) \leq \delta(b-a) .
$$

Let us treat now the case when $f$ is not continuous: we will follow the ideas in 18 .

Lemma 65. Let $f:[a, b] \rightarrow K$ be a strictly increasing definable function. Then, $f(x)$ has a continuous definable inverse; that is, there exists a continuous, nondecreasing, definable function $F$ defined on $[f(a), f(b)]$, such that $F(f(x))=x$ for every $x \in[a, b]$.

Proof. Define $F(y):=\sup \{t: f(t) \leq y\}$.

Lemma 66. Let $\mathbb{K}$ be unrestrained and $a<b \in K$. Let $f:[a, b] \rightarrow K$ be a nondecreasing definable function. Let $E$ be the set of $x \in[a, b]$ such that either $f^{\prime}$ does not exists or it is infinite. Then, $\mu(E)=0$.

Proof. By replacing $f(x)$ with $f(x)+x$, w.l.o.g. we can assume that $f$ is strictly increasing. Thus, we can apply Lemma 65. let $F$ be defined there. By lemmas 64 and 63, $F^{\prime}$ exists and is finite outside a definable set of measure 0. Given $x \neq y \in$ $[a, b]$, we write

$$
\frac{f(y)-f(x)}{y-x}=\left(\frac{F(f(y))-F(f(x))}{f(y)-f(x)}\right)^{-1} .
$$

Thus, if $f$ is continuous at $x$ and $F^{\prime}(x)$ exists, we have that $f^{\prime}(x)=1 / F\left(f^{\prime}(x)\right) \in$ $K \cup\{+\infty\}$. However, by Lemma 39. the set of discontinuity points of $f$ is at most pseudo-enumerable, and a fortiori of measure 0 , and by Lemma 63 . $f^{\prime}(x)<+\infty$ outside a set of measure 0 .

Corollary 67. Let $\mathbb{K}$ be unrestrained. Let $f: K \rightarrow K$ be a definable monotone function. Let $E$ be the set of $x \in K$ such that $f^{\prime}(x)$ does not exists or is infinite. Then, $\mu(E)=0$, and therefore $E$ has empty interior.

7.4. Other problems. Lest the reader thinks the transfer from the real case to the definably complete one is always automatic, we will conclude with an open problem and recall some counterexamples.

Conjecture 68 (Brouwer's Fixed Point). Let $f:[0,1]^{2} \rightarrow[0,1]^{2}$ be a definable continuous function. Then, $f$ has a fixed point, i.e. there exists $c \in[0,1]^{2}$ such that $f(c)=c$.

Fact 69 (Hrushovski, Peterzil [10]). There exists an o-minimal structure $\mathbb{K}$ and a definable $\mathcal{C}^{\infty}$ nonzero function $f: I \rightarrow K$, where $I$ is an open interval around 0 , such that $f(0)=0$ and $f$ satisfies the differential equation

$$
f(x)=x^{2} f^{\prime}(x)+x, \quad f(0)=0, \quad f^{\prime}(0)=11
$$

For every $0<\varepsilon \in \mathbb{R}$ there is no $\mathcal{C}^{1}$ function $f:(-\varepsilon, \varepsilon) \rightarrow \mathbb{R}$ satisfying Equation (7.1).

\footnotetext{
${ }^{1} f$ is the formal power series $\sum_{n \geq 1}(n-1) ! x^{n}$.
} 
Another counterexample to some kind of "transfer principle" for restrained (indeed, locally o-minimal) structures can be found in work by Rennet in [16.

For unrestrained structures, let $\mathcal{R}$ be an expansion of $\langle\mathbb{R},+, \cdot,<, \mathbb{N}\rangle, L$ be its language, $T_{0}$ be the $L$-theory whose models are definably complete structures with a discrete subring, and $T$ be any recursive set of sentences true in $\mathcal{R}$ and extending $T_{0}$. By Gödel's incompleteness theorem, there is a model of $T$ which is not elementarily equivalent to $\mathcal{R}$.

\section{REFERENCES}

[1] A. Bruckner, Differentiation of real functions, AMS, Prividence, Rhode Island USA (1994) CRM mononograph series Vol. 5.

[2] A. Dolich, C. Miller, C. Steinhorn, Structures having o-minimal open core, Trans. Amer. Math. Soc. 362 (2010) 1371-1411

[3] A. Fornasiero, Definably complete structures are not pseudo-enumerable, Arch. Math. Logic 50 (2011) 603-615

[4] A. Fornasiero, Locally o-minimal structures and structures with locally o-minimal open core, Ann. Pure Appl. Logic 164 (2013) 211-229

[5] A. Fornasiero, T. Servi, Definably complete Baire structures, Fund. Math. 209 (2010) $215-241$

[6] P. Hieronymi, Defining the set of integers in expansions of the real field by a closed discrete set, Proc. Amer. Math. Soc. 138 (2010) 2163-2168

[7] P. Hieronymi, Expansions of subfields of the real field by a discrete set, Fund. Math. 215 (2011) 167-175

[8] P. Hieronymi, An analogue of the Baire Category Theorem, J. Symb. Logic (1) 78 (2013) 207-213

[9] P. Hieronymi, M. Tychonievich, Interpreting the projective hierarchy in expansions of the ordered set of real numbers, Proc. Amer. Math. Soc. to appear

[10] E. Hrushovski, I. Peterzil, A question of van den Dries and a theorem of Lipshitz and Robinson; not everything is standard, J. Symb. Logic (1) 72 (2007) 119-122

[11] H. Lebesgue, Sur les fonctions représentables analytiquement, Journal de Mathématiques Pures et Appliquées 1 (1905), 139-216

[12] C. Miller, Expansions of Dense Linear Orders with the Intermediate Value Property, $J$. Symbolic Logic (4) 66 (2001) 1783-1790

[13] C. Miller, Avoiding the projective hierarchy in expansions of the real field by sequences, Proc. Amer. Math. Soc., (5) 134 (2006) 1483-1493

[14] C. Miller, P. Speissegger, Expansions of the real line by open sets: o-minimality and open cores, Fund. Math. 162 (1999), pp. 193-207

[15] J.C. Охтову, Measure and category. A survey of the analogies between topological and measure spaces, Springer-Verlag, New York (1971) GTM Vol.2

[16] A. Rennet, The non axiomatizability of o-minimality, J. Symb. Logic to appear (2013)

[17] F. RIEsz, Sur l'existence de la dérivée des fonctions monotones et sur quelques problèmes qui s'y rattachent, Acta Litt. Sci. Szeged, 5 (1932) 208-221

[18] L.A. Rubel, Differentiability of monotonic functions, Colloq. Math. 10 (1963) 227-279

[19] S.G. Simpson, Subsystems of Second Order Arithmetic, Springer-Verlag, Berlin (1998)

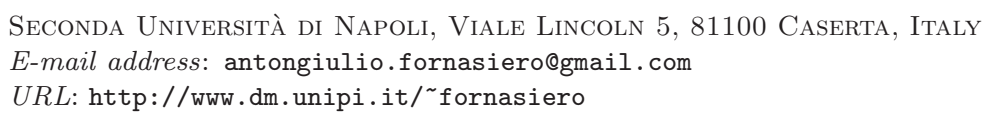

University of Illinois at Urbana-Champaign, Department of Mathematics, 1409 W. Green Street, Urbana, IL 61801, USA

E-mail address: phierony@illinois.edu 